

\title{
Comparison of MRI, MRA, and DSA for Detection of Cerebral Arteriovenous Malformations in Hereditary Hemorrhagic Telangiectasia
}

\author{
(D) M. Vella, (D) M.D. Alexander, (DM.C. Mabray, DD.L. Cooke, (D) M.R. Amans, (DC.M. Glastonbury, (D) H. Kim, (D).W. Wilson,
}

(DD.E. Langston, (DM.B. Conrad, and (D).W. Hetts

\begin{abstract}
BACKGROUND AND PURPOSE: Patients with hereditary hemorrhagic telangiectasia (HHT) have a high prevalence of brain vascular malformations, putting them at risk for brain hemorrhage and other complications. Our aim was to evaluate the relative utility of MR imaging and MRA compared with DSA in detecting cerebral AVMs in the HHT population.
\end{abstract}

MATERIALS AND METHODS: Of 343 consecutive patients evaluated at the University of California, San Francisco HTT Center of Excellence, 63 met the study inclusion criteria: definite or probable hereditary hemorrhagic telangiectasia defined by meeting at least 2 Curacao criteria or positive genetic testing, as well as having at least 1 brain MR imaging and 1 DSA. MRIs were retrospectively reviewed, and the number of AVMs identified was compared with the number of AVMs identified on DSA.

RESULTS: Of 63 patients, 45 (71\%) had AVMs on DSA with a total of 92 AVMs identified. Of those, 24 (26\%) were seen only on DSA; 68 (74\%), on both DSA and MR imaging; and 5 additional lesions were seen only on MR imaging. Of the 92 lesions confirmed on DSA, 49 (53.3\%) were seen on the 3D-T postgadolinium sequence, 52 (56.5\%) were seen on the $2 \mathrm{D}-\mathrm{T} T$ postgadolinium sequence, $35(38.0 \%)$ were seen on the SWI sequence, 24 (26.1\%) were seen on T2 sequence, and $25(27.2 \%)$ were seen on MRA. The sensitivity and specificity of MR imaging as a whole in detecting AVMs then confirmed on DSA were $80.0 \%$ and $94.4 \%$, respectively, and the positive and negative predictive values were $97.3 \%$ and $65.4 \%$, respectively.

CONCLUSIONS: This study reinforces the use of MR imaging as a primary screening tool for cerebral AVMs in patients with hereditary hemorrhagic telangiectasia and suggests that 3D-T postgadolinium and $2 \mathrm{D}-\mathrm{T} 7$ postgadolinium performed at $3 \mathrm{~T}$ are the highest yield sequences.

ABBREVIATION: $\mathrm{HHT}=$ hereditary hemorrhagic telangiectasia

$\mathrm{H}^{2}$

ereditary hemorrhagic telangiectasia (HHT) is a rare, autosomal dominant disease characterized by the formation of mucocutaneous, lung, brain, and visceral organ vascular malformations. ${ }^{1}$ Diagnosis of probable or definite HHT is defined by

\footnotetext{
Received January 30, 2020; accepted after revision March 26.

From the Departments of Radiology and Biomedical Imaging (M.V.), Division of Neurointerventional Radiology (D.L.C., M.R.A., D.E.L., S.W.H.), Division of Diagnostic Neuroradiology (C.M.G.), Division of Interventional Radiology (M.W.W., M.B.C.), Hereditary Hemorrhagic Telangiectasia Center of Excellence (H.K., D.E.L., M.B.C., S.W.H.), and Anesthesia and Perioperative Care (H.K.), University of California, San Francisco, San Francisco, California; Department of Radiology, Division of Interventional Neuroradiology (M.D.A.), University of Utah, Salt Lake City, Utah; and Department of Radiology, Division of Neuroradiology (M.C.M.), University of New Mexico, Albuquerque, New Mexico.

This investigation was supported by the National Institutes of Health under the Ruth L. Kirschstein National Research Service Award T32EB001631 from the National Institute of Biomedical Imaging and Bioengineering, as well as R01EB012031.

Please address correspondence to Steven W. Hetts, MD, Division of Neurointerventional Radiology and HHT Center of Excellence, Department of Radiology and Biomedical Imaging, 505 Parnassus Ave, L-351, San Francisco, CA 94143-0628; e-mail: steven.hetts@ucsf.edu

- Indicates open access to non-subscribers at www.ajnr.org

http://dx.doi.org/10.3174/ajnr.A6549
}

meeting $\geq 2$ of the Curacao criteria or testing positive for known disease-causing mutations, including those in the endoglin (ENG), activin A receptor like type 1 (ACVRL1), and SMAD family member 4 (SMAD4) genes. ${ }^{1,2}$ Up to $23 \%$ of patients with HHT develop cerebral vascular malformations, including brain arteriovenous malformations, cavernous malformations, developmental venous anomalies, capillary telangiectasias, vein of Galen malformations, arteriovenous fistulas, capillary vascular malformations, and transitional or mixed malformations. ${ }^{3-6}$ Most of these lesions are cerebral AVMs, which have the risk of potential rupture. ${ }^{4}$ Studies have reported the risk of rupture in patients with HHT from $0.4 \%$ to $1.3 \%$ per year per patient or $0.3 \%-0.7 \%$ per year per lesion. ${ }^{7,8}$ Given this risk, current guidelines recommend screening with cerebral MR imaging for all adults with possible or definite HHT and all children with possible or definite HHT within the first 6 months of life. ${ }^{1,9}$ These same guidelines suggest using sequences with and without contrast as well as blood-sensitive sequences but provide no further specific recommendations as to which sequences are of the greatest utility. ${ }^{1,9}$ 
While studies have proposed that specific MR images may be beneficial in evaluating the presence of AVMs in non-HHT populations, there are limited data demonstrating the specific utility of these sequences in screening for AVMs in patients with HHT. ${ }^{10-12}$ Because the HHT population is undergoing screening to detect

\section{Table 1: Demographics of patients included in the study}

\begin{tabular}{ll}
\hline \multicolumn{1}{c}{ Demographics } & \\
\hline Total No. of patients & $n=63$ \\
Female sex & $41 / 63(65 \%)$ \\
Age at time of MR imaging (mean) (yr) & $36 \pm 20$ \\
Curacao score & \\
1 & $1 / 63(2 \%)^{\mathrm{a}}$ \\
2 & $18 / 63(29 \%)$ \\
3 & $20 / 63(32 \%)$ \\
4 & $24 / 63(38 \%)$ \\
HHT-causing mutation & \\
ENG & $23 / 63(37 \%)$ \\
ACVRL1 & $5 / 63(8 \%)$ \\
Variant of unknown significance & $2 / 63(3 \%)$ \\
Family member positive & $6 / 63(10 \%)$ \\
Negative genetic testing & $9 / 63(14 \%)$ \\
No genetic testing available & $18 / 63(29 \%)$ \\
Neurologic symptoms & \\
Present & $50 / 63(79 \%)$ \\
Absent & $13 / 63(21 \%)$ \\
\hline a Genetically confirmed HHT
\end{tabular}

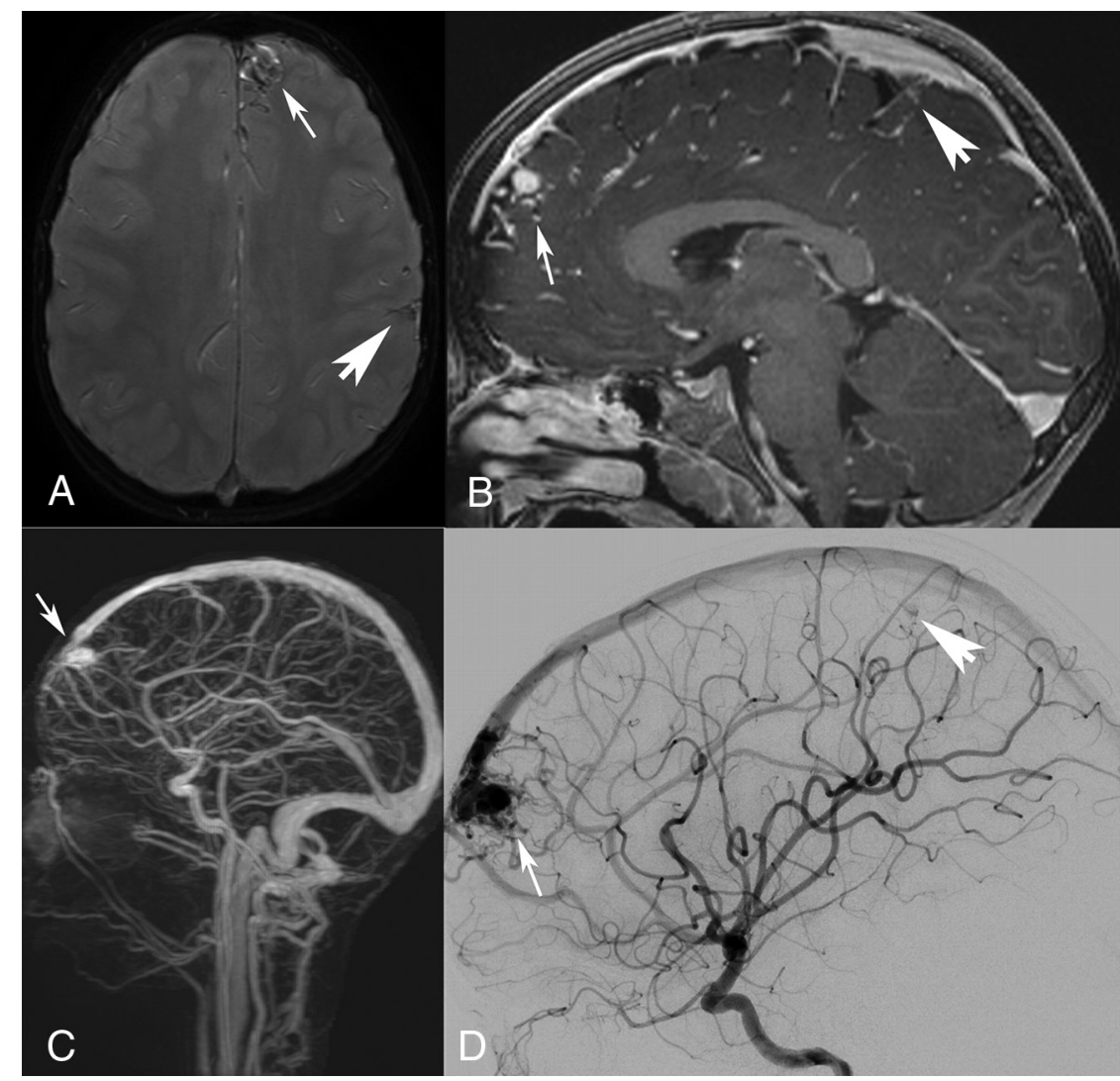

FIG 1. Multiple brain AVMs in HHT: positive on MR imaging, MRA, and DSA. A 5-year-old boy with a frontopolar cerebral AVM (white arrow) demonstrated on SWI MR imaging $(A)$, 3D postgadolinium $\Pi$ $(B)$, time-resolved dynamic contrast-enhanced MRA (C), and DSA (D). A smaller AVM (white arrowhead) is suggested by hypointensity on SWI $(A)$, enhancement on $3 D$ postgadolinium $\Pi(B)$, and enhancement and subtle arteriovenous shunting on DSA (D). The smaller AVM is not seen on MRA (C).
AVMs before they become clinically apparent, the AVMs this screening test seeks to find may often be smaller or have different characteristics compared with those seen on diagnostic examinations of symptomatic patients with AVMs in the general population. ${ }^{1,4}$ We, therefore, sought to evaluate the relative utility of diagnostic screening MR imaging and MRA as well as the utility of specific MR imaging sequences compared with the criterion standard of DSA in detecting cerebral AVMs within the HHT population.

\section{MATERIALS AND METHODS}

This retrospective study was approved by the institutional review board and performed in compliance with Health Insurance Portability and Accountability Act. All patients evaluated at the University of California, San Francisco HHT Center of Excellence as of January 2017 were screened for inclusion in this study $(n=343)$. The medical charts of these patients were retrospectively reviewed, and demographic data including age, sex, Curacao criteria, genetic testing results, and the presence of patient-reported neurologic symptoms were collected, along with information on cerebral imaging to evaluate inclusion in this study.

Inclusion criteria for the study were probable or definite HHT as defined by meeting at least 2 Curacao criteria or positive genetic testing as well as having at least 1 brain MR imaging and 1 cerebral DSA with images available for viewing on our PACS. Of the 343 patients reviewed, 246 met the study definition of probable or definite HHT, and of those, 63 patients had both sets of imaging available for our review and therefore met the full inclusion criteria.

All available MR imaging and MRA images for these 63 patients were retrospectively reviewed by a fellowship-trained neuroradiologist (M.D.A.), a subset of the studies (38 total) was reviewed by a second fellowship-trained neuroradiologist (M.C.M.), and any discrepancies between the 2 readers were adjudicated by a third fellowship-trained neuroradiologist (S.W.H.). All readers were blinded to the official reports. These readers evaluated each MR imaging study as a whole and recorded the total number of AVMs identified for each patient as well as on which sequences the AVMs were visible (3D-T1-weighted postgadolinium, 2D-T1-weighted postgadolinium, MRA, T2-weighted, SWI). Because these MRIs were performed at many different institutions (with patients later being referred to University of California, San Francisco) and on different types and field strengths (0.7T, $1.16 \mathrm{~T}, 1.5 \mathrm{~T}$, and $3 \mathrm{~T}$ ) of MR imaging machines, there was no standardized set of sequences for the evaluated MRIs and MRAs. The cerebral DSA 


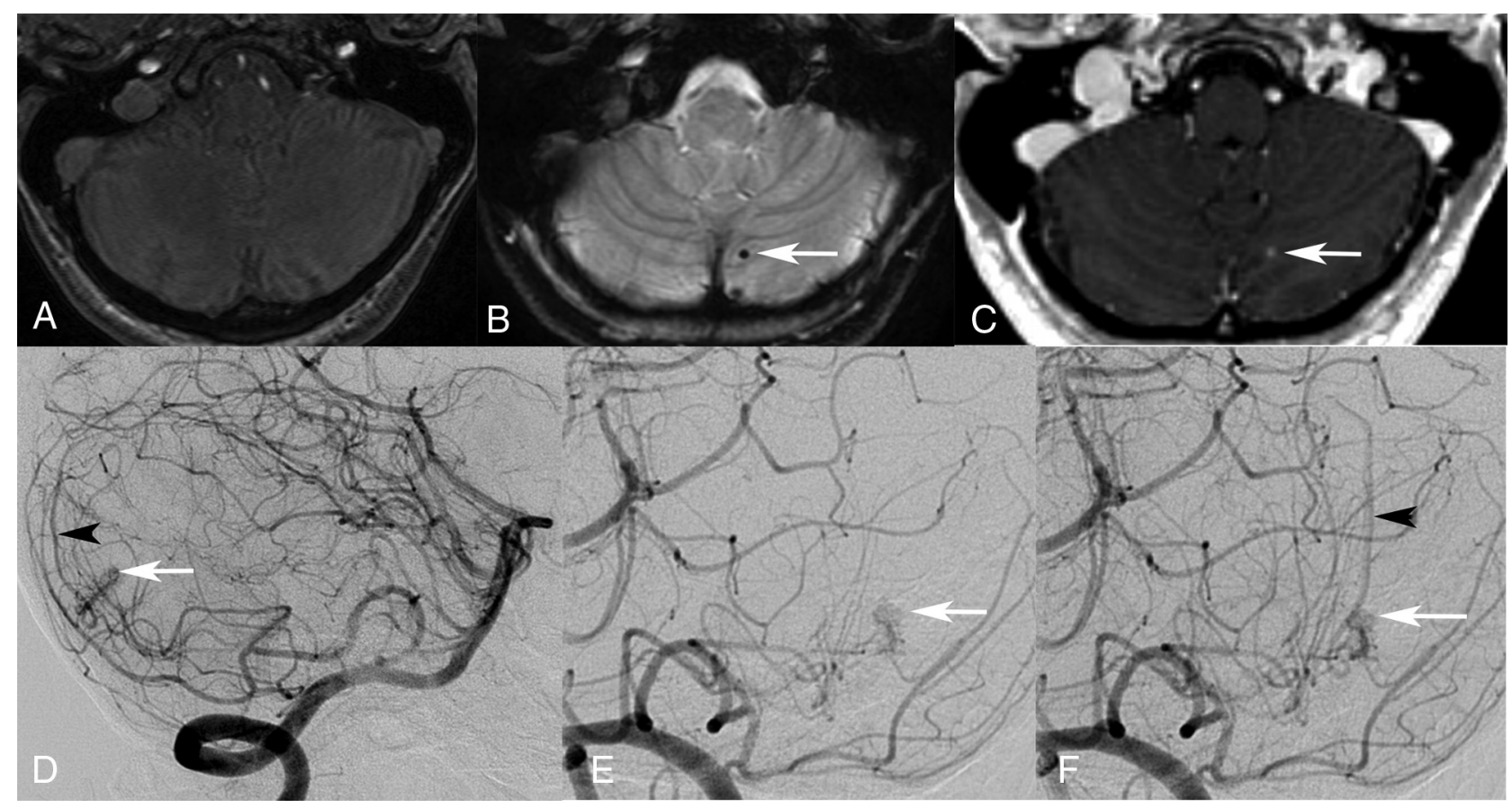

FIG 2. Small cerebellar AVM in HHT: positive on MR imaging, negative on MRA, and positive on DSA. A 49-year-old woman with a cerebellar hemispheric AVM (white arrow) not apparent on TOF-MRA $(A)$ but apparent with microhemorrhage on SWI (B), on 3D postgadolinium Tl (C), and on DSA $(D-F)$. DSA ( $D$ and $F$ ) additionally distinguishes the AVM nidus (white arrow) from the AVM draining vein (black arrowhead).

images for all 63 cases were reviewed by a fellowship-trained neurointerventional radiologist (S.W.H.) who determined and recorded the total numbers of cerebral AVMs visible on DSA.

Once the totals were compiled, true- and false-positive rates of cerebral AVM detection were determined for each identified lesion, with DSA being used as the criterion standard. These data were then used to determine which MR imaging sequences had the highest rates of true-positive cerebral AVM detection. In addition, the sensitivity, specificity, and positive and negative predictive values of MR imaging as a whole (including all available sequences with and without gadolinium and including MRA if available) and each sequence individually were calculated. For these calculations, a true-positive screening MR imaging or sequence was considered to be one that showed any possible AVM even if the exact number of AVMs did not match the number seen on cerebral DSA.

\section{RESULTS}

Of the 63 patients evaluated, $65 \%$ were women, and the average age at the time of the evaluated MR imaging was $36 \pm 20$ years, with a range of 1-77 years (Table 1). The Curacao scores ranged from 1 to 4 because some included patients meeting only a single criterion had positive genetic testing. A total of 39 of the 63 patients underwent genetic testing for known HHT-causing gene mutations, the results of which are listed in Table 1. A total of 24 patients had not undergone genetic testing at the time of the study, $6(9 \%)$ of whom had family members who tested positive for known disease-causing mutations. Self-reported neurologic symptoms ranging from headaches, dizziness, and paresthesias to syncope, seizures, transient ischemic attacks, and strokes were seen in $79 \%$ of patients. Two patients had clinically evident AVM rupture on presentation, both of whom were younger than 12 years of age.
Of the 63 patients whose imaging was evaluated, a total of 45 (71\%) were found to have at least 1 cerebral AVM on criterion standard DSA imaging. A total of 92 individual cerebral AVM lesions were identified in these patients' cerebral DSAs. Of the identified lesions, 24 (26\%) were seen only on cerebral DSA, 68 (74\%) were seen on MR imaging and cerebral DSA, and 5 additional lesions were identified only on MR imaging. Examples of these lesions can be seen in Figs 1-4. Table 2 describes the percentage of total lesions seen on MR imaging as a whole as well as each evaluated sequence compared with the total number of lesions seen on MR imaging $(n=73)$ and DSA $(n=92)$. The lesions seen on MR imaging ranged from 2 to $55 \mathrm{~mm}$. The sensitivity and specificity of MR imaging (including all available sequences with and without gadolinium) as a screening technique used to detect at least 1 cerebral AVM, which was then confirmed on criterion standard cerebral DSA, were $80.0 \%$ and $94.4 \%$, respectively, and the positive and negative predictive values were $97.3 \%$ and $65.4 \%$, respectively (Table 3).

Of the 63 MRIs evaluated, 54 (86\%) included postgadolinium imaging, while the other 9 (14\%) were noncontrast only. Table 4 describes additional characteristics of the MRIs performed, including the field strength and frequency of each type of sequence as well as the spatial resolution ranges for each sequence type. Of the 3 studies not performed on a $1.5 \mathrm{~T}$ or $3 \mathrm{~T}$ magnet, 1 was performed on a $0.7 \mathrm{~T}$ magnet, 1 was listed as a $1.16 \mathrm{~T}$ magnet, and 1 had the magnet strength information missing from the DICOM data. The sensitivity, specificity, and positive and negative predictive values of each of the sequences evaluated as well as MR imaging as a whole and MR imaging based on magnet strength are shown in Table 3. 


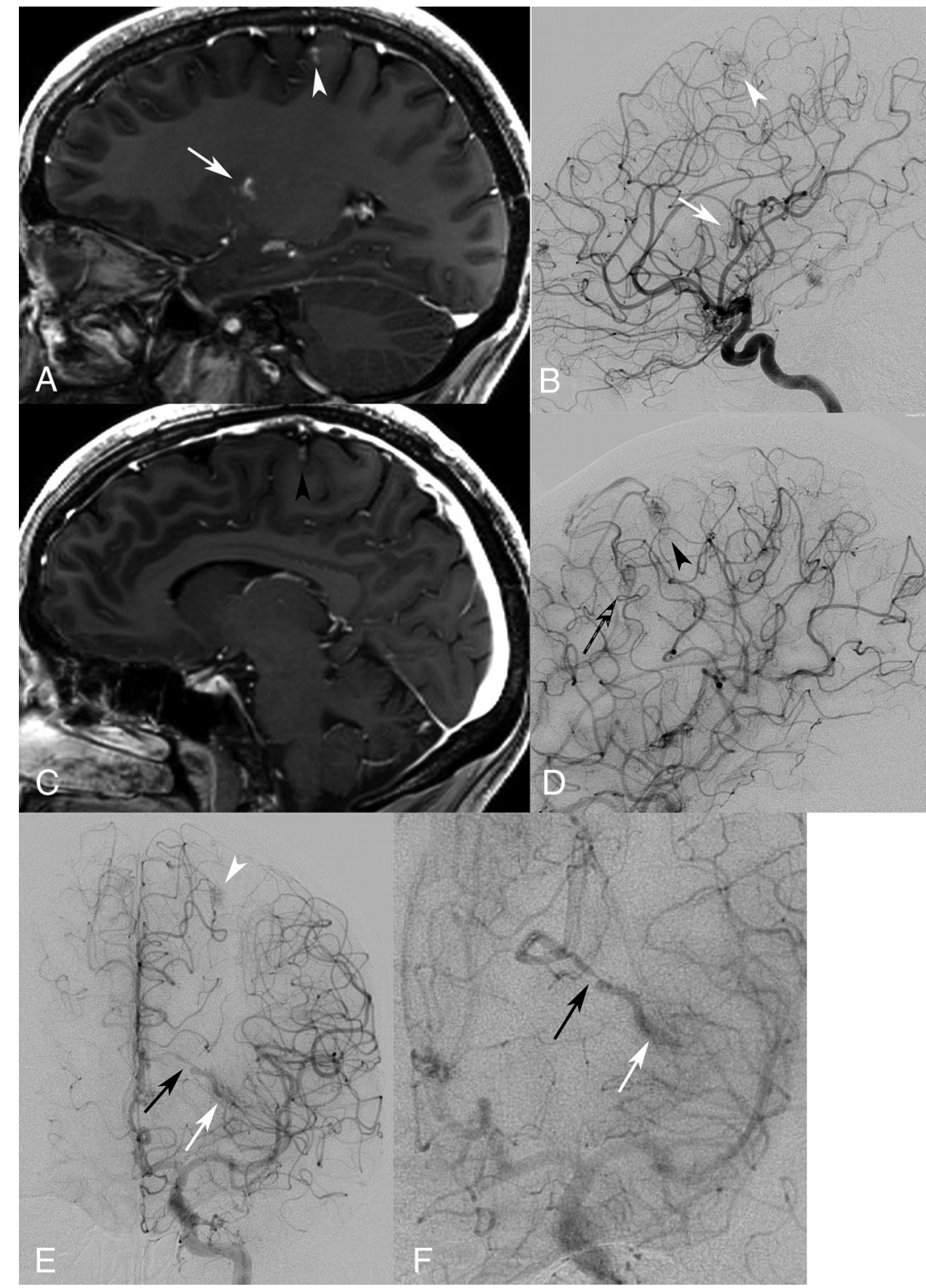

FIG 3. Multiple brain AVMs in HHT: positive on MR imaging, MRA, and DSA. A 23-year-old woman with 2 brain AVMs identified on MRA, 7 brain AVMs identified on MR imaging, and 11 identified on DSA. Selected images demonstrate a left basal ganglia AVM (white arrow) on 3D-postgadolinium $\mathrm{T}(A)$, left ICA lateral DSA (B), left ICA anterior-posterior arterial phase DSA $(E)$, and magnified left ICA anterior-posterior capillary phase DSA $(F)$. Note that the angioarchitecture of the basal ganglia AVM is best seen on DSA ( $E$ and $F$ ), including a high-grade stenosis of the deep draining vein of the AVM (black arrow). Although 3D-postgadolinium $T 1$ image of the right hemisphere (C) demonstrates 1 cortical AVM (black arrowhead), right ICA lateral DSA (D) demonstrates 2 cortical AVMs; the more anterior of these lesions (hashed arrow) was only apparent on DSA.

The sensitivity, specificity, and positive and negative predictive values of each type of MRA can also be found in Table 3. Of note, the 2 postgadoliniumonly MRA studies both yielded falsenegative results, though the statistical significance of this finding is unclear, given the paucity of this type of case. In none of the 50 cases in which MRA was performed were there AVMs that were seen only on the MRA sequence and not on at least 1 additional sequence.

\section{DISCUSSION}

While current guidelines suggest that MR imaging be used as a screening tool for cerebral AVMs in patients with HHT, there are limited data on the accuracy of MR imaging in screening this particular patient population. Our study demonstrates that compared with the criterion standard DSA, MR imaging is relatively sensitive and specific when used to find cerebral AVMs in this population, with a sensitivity of $80.0 \%$, specificity of $94.4 \%$, and a negative predictive value of $65.4 \%$. These data reflect the overall sensitivity and specificity of MR imaging as a screening technique, including all MR imaging sequences evaluated with and without gadolinium. This finding is similar to the reported $80 \%-95 \%$ sensitivity of MR imaging in detecting medium-tolarge cerebral AVMs in the non-HHT population reported in the literature. $^{11-13}$ Our data, on the other hand, suggest that MRA alone (either TOF, postgadolinium, or a combination) as a screening technique is less optimal in the HHT population, with a sensitivity and specificity of $50.0 \%$ and $92.9 \%$, respectively, and a negative predictive value of $41.9 \%$. This finding is further supported by the fact that there were no cases in which a lesion was detected only on an MRA sequence and not on

Of the patients evaluated, 50 had MRAs performed as part of their overall MR imaging examinations. Of these 50 MRAs, 26 (52\%) were performed on a $1.5 \mathrm{~T}$ magnet, 23 (46\%) were performed on a $3 \mathrm{~T}$ magnet, and 1 was reported as being performed on a $1.16 \mathrm{~T}$ magnet. The sensitivity and specificity of MRA alone as a screening technique used to detect at least 1 cerebral AVM, which was then confirmed on criterion standard cerebral DSA, were $50.0 \%$ and $92.9 \%$, respectively, and the positive and negative predictive values were $94.7 \%$ and $41.9 \%$, respectively (Table 3). other sequences as well, suggesting that the addition of MRA to the overall MR imaging did not affect the sensitivity and specificity of MR imaging as a whole. This study supports MR imaging performing well as a screening tool for brain AVMs in the HHT population seen at our Center of Excellence, while MRA should not be used as a sole screening tool for cerebral AVMs in this population.

Our data also suggest that of the MR imaging sequences analyzed, postcontrast imaging sequences were the most useful in detecting cerebral AVMs with 3D-T1 postgadolinium and 2D-T1 


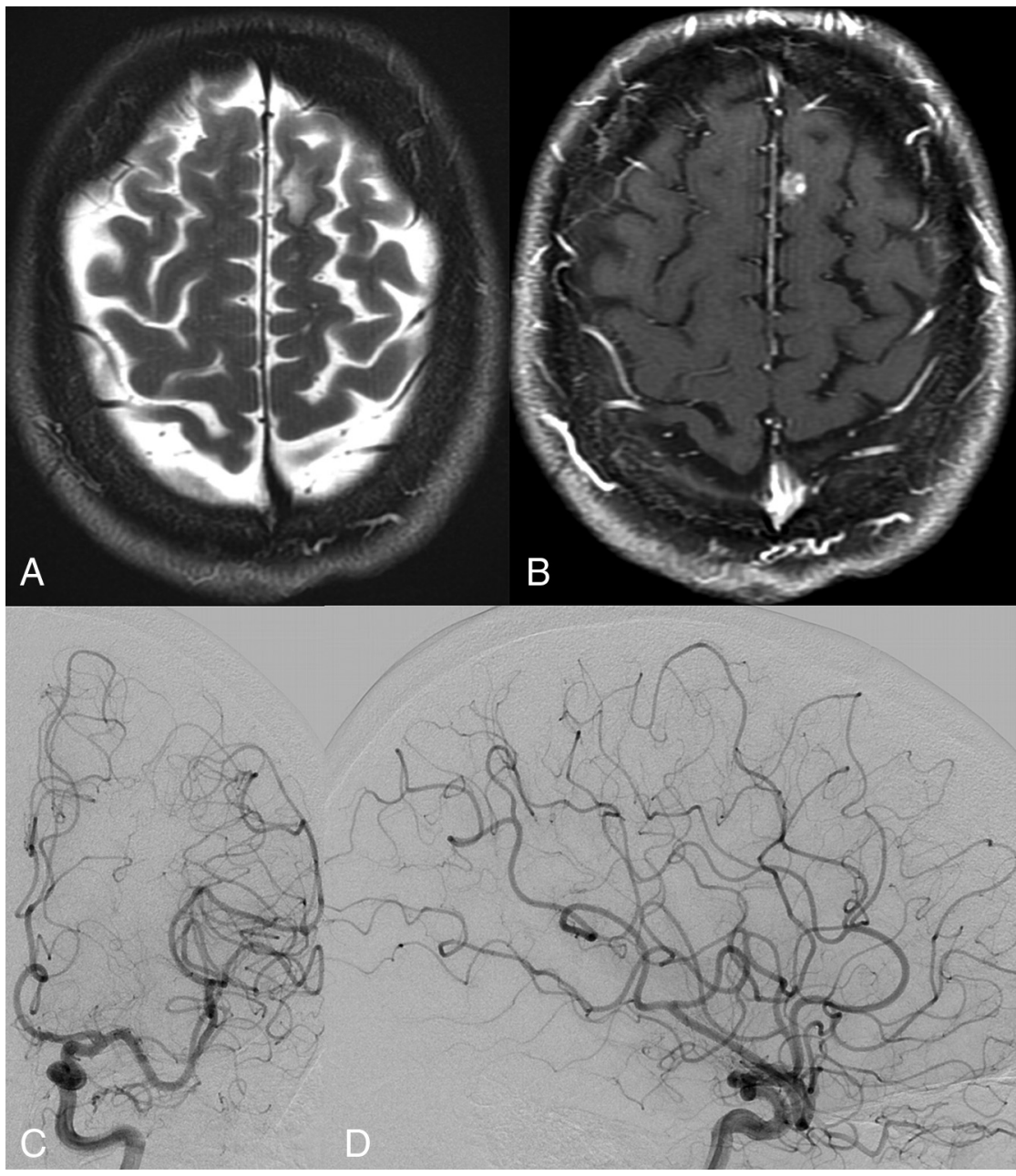

FIG 4. Cerebral vascular lesion in HHT: positive on MR imaging and negative on MRA and DSA. A 72-year-old man previously treated with gamma knife radiosurgery for a left frontal AVM. T2 MR imaging $(A)$ demonstrates gliosis in the superior frontal gyrus but no apparent AVM vessels, postgadolinium 2D-TT MR imaging $(B)$ demonstrates an enhancing lesion in the superior frontal gyrus, and left ICA anterior-posterior (C) and lateral (D) DSA demonstrate no AVM.

Table 2: Number of AVMs seen on each evaluated MR image compared with the total number seen on each technique

\begin{tabular}{lccc}
\hline \multicolumn{1}{c}{ Sequence } & $\begin{array}{c}\text { Total No. of } \\
\text { AVMs Seen on } \\
\text { Given } \\
\text { Sequence }\end{array}$ & $\begin{array}{c}\text { Percentage of all } \\
\text { AVMs Seen on MR } \\
\text { Imaging }(\boldsymbol{n}=\mathbf{7 3})\end{array}$ & $\begin{array}{c}\text { Percentage of } \\
\text { all AVMs Seen } \\
\text { on DSA }(\boldsymbol{n}=\mathbf{9 2})\end{array}$ \\
\hline Total AVMs seen on MR imaging & 73 & $100.0 \%$ & $79.3 \%$ \\
3D-Tl Postgad & 49 & $67.1 \%$ & $53.3 \%$ \\
2D-Tl Postgad & 52 & $71.2 \%$ & $56.5 \%$ \\
SWI & 35 & $47.9 \%$ & $38.0 \%$ \\
T2 & 24 & $32.9 \%$ & $26.1 \%$ \\
MRA & 25 & $34.2 \%$ & $27.2 \%$ \\
\hline
\end{tabular}

Note:-Postgad indicates postgadolinium.

postgadolinium sequences showing up to $56.5 \%$ of lesions later confirmed on cerebral DSA and $71.2 \%$ of those seen on MR imaging. These postgadolinium MR imaging sequences also showed good sensitivity and negative predictive values $(86.2 \%$ and $69.2 \%$, respectively, for 3D-T1 postgadolinium and $75.0 \%$ and $60.9 \%$ respectively, for $2 \mathrm{D}-\mathrm{T} 1$ postgadolinium), which are desirable in a good screening test. While MRA on its own and more specifically
TOF-MRA alone showed poor sensitivity and negative predictive values, suggesting that these sequences would be poor screening tests, the studies that included both TOF and postcontrast MRA showed improved sensitivity and negative predictive values $(70.0 \%$ and $75.0 \%$, respectively, for the combination of TOF and postgadolinium MRA compared with $47.8 \%$ and $25.0 \%$, respectively, for TOF-MRA alone), suggesting that the addition of postgadolinium MRA may improve the utility of this sequence.

In addition, our study suggests that 3T MR imaging is superior to $1.5 \mathrm{~T} \mathrm{MR}$ imaging, with a sensitivity of $85.7 \%$ and specificity of $100.0 \%$ compared with $72.7 \%$ and $92.9 \%$ in $3 \mathrm{~T}$ versus $1.5 \mathrm{~T}$, respectively. While the MRIs performed on other field strengths also appeared to have high sensitivity and specificity, there were too few of these cases to draw firm conclusions.

Our study obviously has several limitations, the most important of which is the heterogeneity of the MR imaging studies that were evaluated. Because these studies came from many different sites, there was variation in the MR imaging magnet strength and the exact sequences performed; thus, we chose to stratify the sequences in the most general way possible rather than by individual manufacturer's sequence type. This heterogeneity of scanners and sequences may, therefore, lead our data to somewhat underestimate the true detection capability of these sequences. Additional studies evaluating a prospective cohort of patients with HHT all scanned on the same scanners with the same standardized protocol of imaging sequences may help to confirm the true sensitivity of each of these sequences in this population.

Whereas MR imaging is a noninvasive test used to screen patients with HHT for brain AVMs, DSA is an invasive test used to confirm findings on MR imaging and go beyond those findings to risk-stratify patients. Predicting the likelihood of future brain AVM rupture is the focus of many academic studies and is of paramount importance to individual patients and their physicians as they decide whether to treat any particular brain AVM. Susceptibilityweighted MR imaging sequences have demonstrated utility in detecting microhemorrhage within sporadic brain AVMs; this 
Table 3: Sensitivity, specificity, and positive and negative predictive values of MR imaging as a whole, individual MR imaging sequences, and MR imaging as a whole at different given magnet strengths for the presence of cerebral $\mathrm{AVM}^{\mathrm{a}}$

\begin{tabular}{|c|c|c|c|c|c|}
\hline & $\begin{array}{l}\text { No. of Positive } \\
\text { Studies }\end{array}$ & Sensitivity & Specificity & $\begin{array}{c}\text { Positive Predictive } \\
\text { Value }\end{array}$ & $\begin{array}{c}\text { Negative Predictive } \\
\text { Value }\end{array}$ \\
\hline \multicolumn{6}{|l|}{ Sequence } \\
\hline MR imaging as a whole ${ }^{b}$ & 37 & $80.0 \%$ & $94.4 \%$ & $97.3 \%$ & $65.4 \%$ \\
\hline 3D-T7 postgadolinium & 25 & $86.2 \%$ & $100.0 \%$ & $100.0 \%$ & $69.2 \%$ \\
\hline 2D-T1 postgadolinium & 27 & $75.0 \%$ & $100.0 \%$ & $100.0 \%$ & $60.9 \%$ \\
\hline $\mathrm{T} 2$ & 20 & $54.1 \%$ & $94.4 \%$ & $95.2 \%$ & $50.0 \%$ \\
\hline SWI & 23 & $51.2 \%$ & $93.8 \%$ & $95.7 \%$ & $41.7 \%$ \\
\hline MRA (any) ${ }^{c}$ & 19 & $50.0 \%$ & $92.9 \%$ & $94.7 \%$ & $41.9 \%$ \\
\hline TOF-MRA only & 11 & $47.8 \%$ & $100.0 \%$ & $100 \%$ & $25.0 \%$ \\
\hline Postgadolinium MRA only & 2 & $0.0 \%$ & NA & NA & $0.0 \%$ \\
\hline TOF and postgadolinium MRA & 8 & $70.0 \%$ & $90.0 \%$ & $87.5 \%$ & $75.0 \%$ \\
\hline \multicolumn{6}{|l|}{ Magnet strength } \\
\hline $1.5 T$ & 17 & $72.7 \%$ & $92.9 \%$ & $94.1 \%$ & $68.4 \%$ \\
\hline 3T & 18 & $85.7 \%$ & $100.0 \%$ & $100.0 \%$ & $50.0 \%$ \\
\hline Other & 2 & $100.0 \%$ & $100.0 \%$ & $100.0 \%$ & $100.0 \%$ \\
\hline
\end{tabular}

Note:-NA indicates not applicable.

${ }^{a}$ A sequence (or MR imaging as a whole) was considered positive if it showed any number of AVMs even if the number did not ultimately match that seen on DSA.

${ }^{b}$ MR imaging as a whole included all available sequences with and without gadolinium evaluated for all patients.

'MRA (any) calculations included TOF and postgadolinium MRA either alone or in combination, depending on what was available for a given study.

Table 4: Characteristics of evaluated MRIs

\begin{tabular}{lcc}
\hline \multicolumn{1}{c}{ Strength/Sequence } & $\begin{array}{c}\text { No. of Cases with Given Strength or } \\
\text { Sequence (\% of 63 Total Cases) }\end{array}$ & $\begin{array}{c}\text { Range of Spatial } \\
\text { Resolution }\end{array}$ \\
\hline Magnet strength & $36(57.1 \%)$ & \\
1.5T & $24(38.1 \%)$ & \\
3T & $3(4.8 \%)$ & \\
Other magnet strength & & \\
Sequence & $38(60.3 \%)$ & $0.4-5 \mathrm{~mm}$ \\
3D-T1 Postgadolinium & $50(79.4 \%)$ & $1-5 \mathrm{~mm}$ \\
2D-T1 Postgadolinium & $59(93.7 \%)$ & $1.6-6 \mathrm{~mm}$ \\
SWI & $53(84.1 \%)$ & $1-7.5 \mathrm{~mm}$ \\
T2 & $50(79.4 \%)$ & $0.5-2.6 \mathrm{~mm}$ \\
MRA (any) & $28(44.4 \%)$ & $0.5-2.6 \mathrm{~mm}$ \\
TOF-MRA only & $2(3.2 \%)$ & $1-1.2 \mathrm{~mm}$ \\
Postgadolinium MRA only & $20(31.7 \%)$ & $1-1.4 \mathrm{~mm}$ \\
TOF and postgadolinium MRA & & \\
\hline
\end{tabular}

Detection of such lesions, thus, remains important for clinical decision-making. Some patients and physicians choose treatment even of small unruptured brain AVMs to prevent future cerebral hemorrhage, particularly if there are angioarchitectural high-risk features, lesional microhemorrhage, genetic predisposition, or a family history of brain AVM hemorrhage.

\section{CONCLUSIONS}

This study reinforces the use of MR imaging as a primary screening tool for cerebral AVMs in patients with HHT and suggests that a combination of post-

finding is a predictor of future AVM rupture. ${ }^{14}$ DSA can demonstrate detailed angioarchitectural features of AVMs that connote a higher risk of future rupture that are often not discernable on MR imaging, including feeding artery aneurysms, nidal aneurysms, and venous outflow stenosis. ${ }^{15,16}$

Our practice has been to perform DSA on patients with HHT who have either a brain hemorrhage or a potential brain AVM identified on screening MR imaging. Thus, another fundamental limitation of our study is that DSA is typically performed on patients who have a suspicious finding on MR imaging or MRA, not on patients who have normal MR imaging and MRA findings, thus likely undercounting the number of potential cerebral vascular malformations identifiable by DSA. There are, of course, small neurovascular lesions that may not be apparent on brain MR imaging but can be detected on DSA. In the HHT population, it would be expected that many of these very small malformations would be capillary vascular malformations that do not have arteriovenous shunting as opposed to nidus-type AVMs that do, by definition, have arteriovenous shunting. Although capillary lesions likely have a lower risk of hemorrhage than shunting lesions, the rupture rate of small shunting AVMs remains unknown. gadolinium sequences, specifically $3 \mathrm{D}-\mathrm{T} 1$ postgadolinium and $2 \mathrm{D}$ $\mathrm{T} 1$ postgadolinium, has the highest yield for brain AVM detection in patients with HHT and that MRA, and in particular TOF-MRA, alone is not sensitive enough to be used as a sole screening tool.

Disclosures: Maya Vella_RELATED: Grant: Ruth L. Kirschstein National Research Service Award T32EB001631, Comments: I am currently pursuing a T32 Research Fellowship year; therefore, any work I publish this year is funded by my salary provided by the T32 grant; UNRELATED: Employment: Radiology Resident Physician at University of California, San Francisco. Marc C. Mabray-UNRELATED: Grants/ Grants Pending: National Institutes of Health/NIH.* Matthew R. AmansUNRELATED: Consultancy: Covidien, Comments: Pipeline proctor. Christine $M$ Glastonbury-UNRELATED: Royalties: Amirsys-Elsevier, Comments: royalties from books and book chapters. Helen Kim-RELATED: Grant: National Institutes of Health; UNRELATED: Consultancy: Recursion Pharmaceuticals; Grants/Grants Pending: National Institutes of Health. Steven W. Hetts-RELATED: Grant: National Institute of Biomedical Imaging and Bioengineering*; UNRELATED: Consultancy, MicroVention Terumo, Route 92 Medical, and Imperative Medical Comments: CEC member for unrelated stroke treatment clinical trials; Core imaging laboratory for clinical trials: Stryker Neurovascular.* *Money paid to the institution.

\section{REFERENCES}

1. Faughnan ME, Palda VA, Garcia-Tsao G, et al; HHT Foundation International Guidelines Working Group. International guidelines 
for the diagnosis and management of hereditary haemorrhagic telangiectasia. J Med Genet 2011;48:73-87 CrossRef Medline

2. Shovlin CL, Guttmacher AE, Buscarini E, et al. Diagnostic criteria for hereditary hemorrhagic telangiectasia (Rendu-Osler-Weber syndrome). Am J Med Genet 2000;91:66-67 CrossRef Medline

3. Maher CO, Piepgras DG, Brown RD Jr, et al. Cerebrovascular manifestations in 321 cases of hereditary hemorrhagic telangiectasia. Stroke 2001;32:877-82 CrossRef Medline

4. Fulbright RK, Chaloupka JC, Putman CM, et al. MR of hereditary hemorrhagic telangiectasia: prevalence and spectrum of cerebrovascular malformations AJNR Am J Neuroradiol 1998;19:477-84 Medline

5. Krings T, Kim H, Power S, et al; Brain Vascular Malformation Consortium HHT Investigator Group. Neurovascular manifestations in hereditary hemorrhagic telangiectasia: imaging features and genotype-phenotype correlations. AJNR Am J Neuroradiol 2015;36:863-70 CrossRef Medline

6. Brinjikji W, Iyer VN, Lanzino G, et al. Natural history of brain capillary vascular malformations in hereditary hemorrhagic telangiectasia patients. J Neurointerv Surg 2017;9:26-28 CrossRef Medline

7. Yang W, Liu A, Hung AL, et al. Lower risk of intracranial arteriovenous malformation hemorrhage in patients with hereditary hemorrhagic telangiectasia. Neurosurgery 2016;78:684-93 CrossRef Medline

8. Willemse RB, Mager JJ, Westermann CJ, et al. Bleeding risk of cerebrovascular malformations in hereditary hemorrhagic telangiectasia. J Neurosurg 2000;92:779-84 CrossRef Medline

9. Easey AJ, Wallace GM, Hughes JM, et al. Should asymptomatic patients with hereditary haemorrhagic telangiectasia (HHT) be screened for cerebral vascular malformations? Data from 22,061 years of HHT patient life. J Neurol Neurosurg Psychiatry 2003;74:74348 CrossRef Medline

10. Sommer C, Mullges W, Ringelstein EB. Noninvasive assessment of intracranial fistulas and other small arteriovenous malformations. Neurosurgery 1992;30:522-28 CrossRef Medline

11. Mori H, Aoki S, Okubo T, et al. Two-dimensional thick-slice MR digital subtraction angiography in the assessment of small to medium-size intracranial arteriovenous malformations. Neuroradiology 2003;45:27-33 CrossRef Medline

12. Mukherji SK, Quisling RG, Kubilis PS, et al. Intracranial arteriovenous malformations: quantitative analysis of magnitude contrast MR angiography versus gradient-echo MR imaging versus conventional angiography. Radiology 1995;196:187-93 CrossRef Medline

13. Gauvrit JY, Oppenheim C, Nataf F, et al. Three-dimensional dynamic magnetic resonance angiography for the evaluation of radiosurgically treated cerebral arteriovenous malformations. Eur Radiology 2006; 16:583-91 CrossRef Medline

14. Guo Y, Saunders T, Su H, et al. Silent intralesional microhemorrhage as a risk factor for brain arteriovenous malformation rupture. Stroke 2012;43:1240-46 CrossRef Medline

15. Hetts SW, Cooke DL, Nelson J, et al. Influence of patient age on angioarchitecture of brain arteriovenous malformations. AJNR Am J Neuroradiol 2014;35:1376-80 CrossRef Medline

16. Alexander MD, Cooke DL, Nelson J, et al. Association between venous angioarchitectural features of sporadic brain arteriovenous malformations and intracranial hemorrhage. AJNR Am J Neuroradiol 2015;36:949-52 CrossRef Medline 